\title{
Coping with occupational stress: the role of optimism and coping flexibility
}

\author{
This article was published in the following Dove Press journal: \\ Psychology Research and Behavior Management \\ 18 April 2016 \\ Number of times this article has been viewed
}

\section{Daniel J Reed}

Department of Psychology, Sheffield Hallam University, Collegiate Campus, Sheffield, UK
Correspondence: Daniel J Reed Department of Psychology, Sheffield Hallam University, Collegiate Campus, Collegiate Crescent, Sheffield SIO 2BQ, UK

Tel +447909828 676

Email danielreedtt@gmail.com
Abstract: The current study aimed at measuring whether coping flexibility is a reliable and valid construct in a UK sample and subsequently investigating the association between coping flexibility, optimism, and psychological health - measured by perceived stress and life satisfaction. A UK university undergraduate student sample ( $\mathrm{N}=95)$ completed an online questionnaire. The study is among the first to examine the validity and reliability of the English version of a scale measuring coping flexibility in a Western population and is also the first to investigate the association between optimism and coping flexibility. The results revealed that the scale had good reliability overall; however, factor analysis revealed no support for the existing two-factor structure of the scale. Coping flexibility and optimism were found to be strongly correlated, and hierarchical regression analyses revealed that the interaction between them predicted a large proportion of the variance in both perceived stress and life satisfaction. In addition, structural equation modeling revealed that optimism completely mediated the relationship between coping flexibility and both perceived stress and life satisfaction. The findings add to the occupational stress literature to further our understanding of how optimism is important in psychological health. Furthermore, given that optimism is a personality trait, and consequently relatively stable, the study also provides preliminary support for the potential of targeting coping flexibility to improve psychological health in Western populations. These findings must be replicated, and further analyses of the English version of the Coping Flexibility Scale are needed.

Keywords: stress management, psychological health, student stress, life satisfaction, organizations

\section{Introduction}

\section{The impact of stress and coping strategies}

The occupational literature defines work-related stress as the adverse reaction people experience to excessive pressure or other types of work-related demands placed on them. ${ }^{1,2}$ Much of the literature on occupational stress emphasizes its adverse effects on employees' health and their organizations. ${ }^{1}$ According to the statistical data released by the UK Health and Safety Executive in 2013/2014, work-related stress, depression, or anxiety accounted for 39\% (487,000 cases) of all work-related illnesses, with 11.3 million work days being lost. ${ }^{3}$ This negating of the impact of occupational stress is very closely related to major psychological problems such as depression and has been noted among a large proportion of UK university students. ${ }^{4,5}$

It is widely accepted that research around coping is vital in order to increase understanding of the impacts of stress on people. ${ }^{6}$ Coping has been defined as comprising "efforts to prevent or diminish threat, harm, and loss, or to reduce associated 
distress". ${ }^{7}$ The transactional theory, developed by Lazarus and Folkman, ${ }^{2}$ is a prominent theory of coping. It consists of three central elements: stress, appraisal, and coping. Stress results from the appraisal of demands as being beyond the resources of the person. Coping involves using both cognitive and behavioral efforts to manage this stress. The original transactional theory outlined two key coping functions: problemcentered coping is directed toward dealing with the conditions triggering stress, while emotion-centered coping is directed toward regulating accompanying emotions. ${ }^{2}$ An important aspect of the theory is its view of coping as a goal-directed process, which has received empirical support from numerous studies. ${ }^{8}$ Furthermore, coping strategy has been utilized in cognitive-behavioral interventions. ${ }^{9}$ For these reasons, it may be inferred that coping can be developed and improved in order to help people handle stress more effectively.

However, the transactional theory has also been criticized for being overly broad, resulting in difficulty around placing such a wide range of coping types into two general categories. ${ }^{7}$ For example, walking away from a conflict could be described as either an emotion-focused coping style, through its tendency to calm emotions down, or a problem-focused coping style, through its leading to taking time to think. ${ }^{10}$ Furthermore, despite more recent attempts to distinguish between and group different strategies, there has been little agreement on how to conceptualize or measure ways of coping. ${ }^{6}$ Bonanno and Burton ${ }^{11}$ argue that most research has been undertaken with the assumption that coping strategies are consistently either beneficial or maladaptive. This is supported by Folkman and Moskowitz, ${ }^{9}$ who highlighted that the context of a stressful event is highly relevant, and a coping strategy can be effective in one situation but not in another. In attempts to address this, it has been put forward that one important aspect of coping is flexibility, such as using different coping strategies depending on the stressful situations. ${ }^{12,13}$

\section{Coping flexibility}

Cheng's ${ }^{12}$ goodness-of-fit approach was one of the first to discuss ways of examining coping flexibility as a construct. Interventions based on the goodness-of-fit approach have been successfully implemented. ${ }^{14}$ Kato $^{15}$ defines coping flexibility as "the ability to discontinue an ineffective coping strategy and produce and implement an alternative coping strategy" (p 263). The theory builds upon transactional theory, more specifically incorporating aspects of the revised model, ${ }^{16}$ in which coping is viewed as a process through which individuals continually attempt to resolve their problems. Unlike Cheng's ${ }^{12}$ goodness-of-fit approach, Kato ${ }^{15}$ considered the effectiveness of a strategy and treated coping as an ongoing process that requires continuous monitoring.

Kato's ${ }^{15}$ definition has two major processes: evaluation coping and adaptive coping. Evaluation coping is the process of stopping an ineffective coping strategy, and it includes the importance of understanding one's environment, monitoring and evaluating the outcomes of coping, and, if outcomes are unfavorable, then eventually stopping the ineffective coping strategy. ${ }^{15}$ Adaptive coping, on the other hand, refers to the process of creating and starting alternative strategies that are available. If adaptive coping does not result in a favorable outcome, then the whole process of evaluation-adaptive coping is repeated. Kato ${ }^{15}$ developed the Coping Flexibility Scale (CFS) - a self-report measure of the construct. The scale has been reported to have strong reliability in an Asian sample. ${ }^{17}$ Studies using the CFS have found coping flexibility to be a strong predictor of psychological health in students and employees, which negatively correlates with anxiety, depression, and stress. ${ }^{15,17}$ The implication here is that people unable to cope with stressful situations have poor coping flexibility.

Having an all-encompassing method and measure for all types of coping makes it possible to generalize the results, allowing coping flexibility to be studied beyond the typical areas of coping and stress. Kato's ${ }^{15}$ model is also built upon existing theories from both the coping and self-regulation literature, known as the Dual-process Model of Coping, ${ }^{18}$ as well as the Social Problem-solving Theory. ${ }^{19}$ The CFS, therefore, appears to be the most reliable instrument for assessing coping flexibility. Despite this observation, the reported studies that have employed the CFS have mainly been done with Asian samples, and the validity and reliability of the English version of the CFS within a Western sample need to be tested.

\section{Optimism}

Optimism is an individual difference variable that reflects the extent to which people hold generalized favorable expectancies for their future. Higher levels of optimism have been related to better subjective well-being in times of adversity or difficulty. ${ }^{20}$ Consistent with such findings, optimism has been linked to higher levels of engagement coping and lower levels of avoidance, or disengagement, coping. ${ }^{21}$ There are two key conceptualizations of optimism: explanatory style, which is a product of the "Learned Helplessness Theory"; 22 and dispositional optimism, which is viewed as a relatively constant aspect of personality - which is the type of optimism the present study is concerned with. ${ }^{20}$ 
Optimism is measured by asking people whether they expect outcomes in their lives to be good or bad. This approach is reflected in the Life Orientation Test (LOT), ${ }^{23}$ and its revised predecessor (LOT-R), ${ }^{24}$ both of which are self-report measures. Studies using the constructs have examined the relationship between optimism and stress involving both, students and employees. Brissette et $\mathrm{al}^{25}$ reported that higher levels of optimism among students adjusting to their first semester of university were associated with smaller increases in both stress and depression. Similarly, De Terte et $\mathrm{a}^{26}$ found a strong negative correlation between optimism and stress in police officers. Hence, optimism is assumed to predict resilience against stressful life events in both students and employees.

One of the important processes through which optimism results in lower levels of stress is demonstrated by the differences in how optimists and pessimists cope with stress. It has been argued that optimists generally have an engaged approach to life, appraising stressful events more positively and using more approach coping strategies, while pessimists have a more disengaged approach and use more avoidance strategies. ${ }^{7,21}$ A meta-analysis of optimism and coping by Nes and Segerstrom ${ }^{27}$ supported this view. They found that optimism was positively associated with broad measures of approach coping and with problem-focused coping. Furthermore, optimists were responsive to the sort of stressor that was being confronted. Optimism predicted more problem-focused coping with controllable stressors (eg, academic demands) and more emotion-focused coping with uncontrollable stressors (eg, trauma). Alternatively, pessimism was related to both subsets of avoidance coping: problem-focused disengagement (eg, behavioral disengagement) and emotion-focused disengagement (eg, denial and wishful thinking).

\section{Life satisfaction}

A secondary objective of the current study is to explore the impact that coping flexibility and optimism have on wellbeing. This is in line with Kato's ${ }^{15}$ assertion that "the purpose of research on coping is to sustain or enhance well-being as well as reduce distress" ( $p$ 270). Life satisfaction is an aspect of well-being, which is a conscious cognitive judgment of one's life, in which the criteria for judgment are selected by the person. ${ }^{28}$ The present study will thus use the Satisfaction with Life Scale (SWLS) to measure subjective well-being. ${ }^{28}$ Researchers have reported a strong, negative correlation between life satisfaction and perceived stress. ${ }^{17}$ Furthermore, they reported that life satisfaction was also correlated with coping flexibility, as measured by the CFS. Other studies have suggested that individuals high in optimism have higher levels of well-being..$^{29}$ For example, work by Chang ${ }^{30}$ informs that optimism in undergraduate students correlates highly with life satisfaction. Conversano et $\mathrm{a}^{31}$ have suggested that it is possible to avoid or reduce the negative psychological effects after not achieving a goal by being adaptive and finding alternative goals. These two elements are significantly connected with both coping flexibility and optimism and should lead to higher life satisfaction.

\section{The interaction between coping flexibility and optimism}

Nes and Segerstrom's ${ }^{27}$ study showed a connection between optimism and coping flexibility: optimistic individuals were able to change coping strategies depending on the situation. In addition, a study by Pavlova and Silbereisen ${ }^{32}$ on the impact of optimism in occupational uncertainty found that optimism predicted an increase in approach strategies only under favorable conditions in the labor market. What these studies inform is that optimists are also better at tailoring their coping responses to available opportunities. While demonstrating the close relationship between coping flexibility and optimism, such studies have supported the goodness-of-fit approach to coping flexibility. ${ }^{12}$ These studies have argued that optimistic individuals change their coping strategy depending on the situation (ie, whether it is controllable).

Not many studies have examined personality and coping flexibility using the CFS, as this is a relatively new construct. A notable exception is a recent study by $\mathrm{Ng}$ et a ${ }^{17}$ that investigated the relationship between narcissism and perceived stress. The study found that coping flexibility mediates the relationship between subtypes of narcissism, with grandiose narcissists being more flexible in coping with stress than vulnerable narcissists. An explanation offered for the observation was that grandiose narcissism is more related to approach motivation, whereas vulnerable narcissism is more tied to avoidance motivation. ${ }^{33}$ The possibility therefore is that, there could be a similar strong relationship between optimism and coping flexibility, as the approach-oriented coping seen in optimistic individuals should allow them to cope with stress more flexibly than avoidance-oriented pessimistic individuals. In effect, optimism should facilitate flexible coping by directing individuals to focus on the positive rewards, rather than the risks of adopting alternative coping strategies. Other support for this comes from evidence of greater coping flexibility in individuals who are better at taking different perspectives in the stress appraisal process ${ }^{13}$ more positive 
beliefs and states allowing individuals to consider negative information appropriately and thus make better coping-based decisions $;^{34}$ and the ability to shift focus from distressing issues and shift one's focus toward the future, resulting in greater coping flexibility. ${ }^{35}$

In summary, the evidence discussed so far suggests that there is a strong relationship between coping flexibility and optimism, with both facilitating effective coping strategies. The evidence and assumptions for how coping flexibility will interact with optimism suggest that flexibility will involve more than the goodness-of-fit approach. Kato's ${ }^{15}$ approach will, therefore, appropriately capture the contents of coping flexibility more efficiently, and further help with our understanding of how optimists effectively handle coping.

\section{Overview of the present research}

The study had two aims: first, to test the validity and reliability of the English version of the CFS within a UK sample using factor analysis; and second, to investigate the association between coping flexibility and optimism and further examine their association with psychological health, measured by perceived stress and life satisfaction. Four hypotheses are anticipated.

\section{Hypothesis I}

The English version of the CFS will demonstrate high validity and reliability in a UK sample.

\section{Hypothesis 2}

Coping flexibility and optimism will be positively and strongly correlated.

\section{Hypothesis 3}

Coping flexibility and optimism will explain a large proportion of the variance in both perceived stress and life satisfaction.

\section{Hypothesis 4}

Optimism will mediate the relationship between coping flexibility and both perceived stress and life satisfaction.

\section{Methodology}

\section{Participants}

Respondents were $(\mathrm{N}=95)$ undergraduate psychology students (not including eleven students who submitted incomplete questionnaires). They completed an online questionnaire. The majority were first-year students $(87 \%, n=83)$, recruited through a scheme in which they gain research credits as a requirement of the course. All remaining participants were third-year students recruited via convenience sampling $(n=12)$. Participant ages ranged between 18 and 35 years (mean $=19.68)$. Eighty-six percent of participants were female $(n=82)$ and fourteen percent were male $(n=13)$. The nationalities of students available for the sample were as follows: 95\% British, 2\% European, 2\% Asian, 0.5\% African, and $0.5 \%$ North American. The response rate was $25 \%$.

\section{Measures}

The Perceived Stress Scale (PSS) was used to measure perceived stress. ${ }^{36}$ The PSS has ten items that measure the frequency of stress feelings in the past month (eg, "In the last month, how often have you felt nervous and 'stressed?'"). Respondents were asked to respond on a 5-point scale $(1=$ never; $5=$ very often). There are also some positively stated (reverse-scored) items (eg, "In the last month, how often have you felt that things were going your way?"). Cronbach's $\alpha$ in the present study was 0.89 .

The SWLS was used to measure life satisfaction. ${ }^{28}$ The SWLS has five items that measure participants' general satisfaction with life. Sample items were: ("I am satisfied with my life"); with responses on a 7-point scale ( $1=$ strongly disagree; $7=$ strongly agree). Cronbach's $\alpha$ in the present study was 0.89 .

The CFS was used to measure coping flexibility. ${ }^{15}$ The CFS was translated by the original scale makers in the English version of the article, which can be found in the original article's supplement. ${ }^{15}$ The CFS has ten items that measure participants' ability to monitor and discontinue ineffective coping (evaluation coping; eg, "if I feel that I have failed to cope with stress, I change the way in which I deal with stress") and use alternative coping strategies (adaptive coping; eg, "If I have failed to cope with stress, I think of other ways to cope"). Two of the evaluation coping items are reverse-scored (eg, "I only use certain ways to cope with stress"). Participants responded on a 4-point scale (1= not applicable; $4=$ very applicable). Cronbach's $\alpha$ in the present study was 0.81 .

The LOT-R was used to measure optimism. ${ }^{24}$ The LOT-R has six items (plus four filler items) that measure the individual difference in dispositional optimism (eg, "I'm always optimistic about my future"), with three reverse-scored items (eg, "I rarely count on good things happening to me"). Participants responded on a 5 -point scale $(1=$ strongly disagree; $5=$ strongly agree). Higher scores on the LOT-R generally reflect a greater tendency to expect more positive versus negative outcomes. Cronbach's $\alpha$ in the present study was 0.85 . 


\section{Procedure}

The current study was conducted with the approval of the Sheffield Hallam University Ethics Committee. All procedures followed were in accordance with the ethical standards of the British Psychological Society Code of Ethics and Conduct. The self-report measures were completed online using Qualtrics online software. ${ }^{37}$ Ninety-five participants were presented with information about the study. They were informed that participation was voluntary and that they were free to withdraw participation at any time. In addition, all participants were assured of anonymity and confidentiality. Consent was obtained by clicking to proceed after reading the information about the study. Upon completion of the questionnaire, participants were informed again about their right to withdraw, and if happy, they were given the opportunity to submit their answers by clicking the submit button.

\section{Data analyses}

To meet the purpose of the present study, first, the validity and reliability of the English version of the CFS was tested internal consistency reliability, item-total correlation, principle components analysis (PCA), parallel analysis (PA), and confirmatory factor analysis (CFA). Second, the association between the variables was examined using correlation analyses and hierarchical regression analyses. Structural equation modeling (SEM) with bootstrapping was finally used to further examine the proposed mediating effect of optimism on the association between coping flexibility and both perceived stress and life satisfaction. Prior to the analyses, the data were checked to ensure no violations of the assumptions of normality, outliers, and multicollinearity. Both the dependent variables - perceived stress (PSS) and life satisfaction (SWLS) - were normally distributed with no extreme outlier effects. A check of the Tolerance and Variance Inflation Factor showed no issue with multicollinearity. The tolerances were all greater than $0.10^{38}$ and ranged between
0.78 and 0.99 ; Variance Initiation Factors were all below $10^{39}$ and ranged between 1.01 and 1.28.

\section{Results}

Table 1 summarizes the descriptive statistics and correlations between the variables. Regarding the first aim of the study, the Cronbach's $\alpha$ indicated a high reliability for the CFS $(\alpha=0.81)$, which is consistent with Hypothesis 1 . Furthermore, within the CFS, all skewness and kurtosis values were less than the absolute value of 1.0, except for item 9, indicating a normal distribution of scores. Further analysis revealed that one item was not internally consistent with the scale - item 2 had low item-total correlation $(r=0.10)$.

Analysis of the subscales revealed some issues with the construct. While the adaptive coping subscale had good reliability $(\alpha=0.85)$, the evaluation coping subscale had low reliability $(\alpha=0.48)$. The only two reverse-scored items in the CFS are within the evaluation coping subscale, and these were found to have low internal consistency, as measured by itemtotal correlation - item $2(r=0.08)$ and item $7(r=0.19)$.

A PCA was conducted to further test the scale. This initial analysis revealed three components with eigenvalues $>1$, which explained $41.19 \%, 11.93 \%$, and $10.85 \%$ of the variance in the scale, in which the first component $(41.19 \%)$ contained all ten items. In addition, a PA was performed ${ }^{40}$ based on Cota et al' ${ }^{41}$ suggestion, in which 95 th percentile eigenvalues for 1,000 random data sets were used. The PA indicated that a one-factor solution would be appropriate. Thus, the findings do not support the existing two-factor structure of the CFS in an English-speaking Western sample.

A CFA was conducted using Amos 23.0 software (IBM Corporation, Armonk, NY, USA) and using a maximum likelihood method to test the one-factor (1) and two-factor (2) model fit of the scale. Chi-square value for both models was significant (1) $\chi^{2}(d f=35, \mathrm{~N}=95) 111.78, P<0.001$; (2) $\left.\left.\chi^{2}(d f=34, \mathrm{~N}=95) 105.37, P<0.001\right)\right]$, suggesting a lack of fit between the hypothesized model and the data. Furthermore,

Table I Descriptive statistic, Cronbach's $\alpha$, and intercorrelations among variables under study

\begin{tabular}{|c|c|c|c|c|c|c|c|c|c|}
\hline Variable & $\mathbf{M}$ & SD & $\alpha$ & Age & Sex & PSS & SWLS & CFS & LOT-R \\
\hline Age & 19.68 & 2.89 & NA & - & & & & & \\
\hline Sex & $2^{\mathrm{a}}$ & 0.35 & NA & 0 & - & & & & \\
\hline PSS & 3.21 & 0.69 & 0.89 & 0.04 & $0.28^{* *}$ & - & & & \\
\hline SWLS & 4.57 & 1.35 & 0.89 & -0.12 & -0.16 & $-0.58 * *$ & - & & \\
\hline CFS & 2.52 & 0.49 & 0.81 & 0.04 & -0.20 & $-0.39 * *$ & $0.33^{* *}$ & - & \\
\hline LOT-R & 3.00 & 0.77 & 0.85 & 0.06 & -0.10 & $-0.60 * *$ & $0.69 * *$ & $0.45 * *$ & - \\
\hline
\end{tabular}

Notes: $\mathrm{N}=95$. ${ }^{* *} \mathrm{P}<0.0 \mathrm{I}$. Values are given as mean, standard deviations, Cronbach's $\alpha$, and zero-order correlations of study variables. ${ }^{a}$ Mode. Values for sex: male $=1$ and female $=2$.

Abbreviations: CFS, Coping Flexibility Scale; LOT-R, Life Orientation Test-Revised; M, mean; NA, not applicable; PSS, Perceived Stress Scale; SD, standard deviation; SWLS, Satisfaction with Life Scale. 
examination of these indices also showed unacceptable model fit for CFA guidelines, ${ }^{42}$ including the error of approximation (1) root mean square error of approximation [RMSEA] $=0.15$; (2) RMSEA $=0.15]$ and all model fit indices [eg, (1) comparative fit index $[\mathrm{CFI}]=0.78$, goodness-of-fit index $[\mathrm{GFI}]=0.81$, normed fit index $[\mathrm{NFI}]=0.71$; (2) CFI $=0.79$, $\mathrm{GFI}=0.83$, NFI $=0.73$, where the two-factor model was a slightly better fit.

Thus, it appears that the low reliability of the evaluation coping subscale is likely caused by the only two reverse-scored items in the CFS. This could explain the lack of support for the two-factor structure of the CFS. The poor model fit of the one-factor model also corresponds with similar results from previous studies within a Japanese sample, ${ }^{1}$ where the two-factor model was a better fit to the data. These findings must be replicated, and further analyses of the English version of the scale are needed within Western samples.

Nonetheless, considering the strong reliability of the CFS and the high proportion of variance explained by the onefactor version, there is some preliminary support for Hypothesis 1. From the current sample, the author interpreted that a one-factor solution is most appropriate, and for subsequent analyses, the complete ten-item CFS will be used.

The correlational evidence is consistent with previous studies. ${ }^{15,17,30}$ Students with higher optimism and coping flexibility reported both lower perceived stress levels and higher life satisfaction. Importantly, optimism had a stronger relationship with both dependent variables. Perceived stress was also strongly and negatively correlated with life satisfaction, as would be expected. Furthermore, consistent with Hypothesis 2, coping flexibility and optimism were strongly correlated, indicating that further analysis of their interaction is warranted.

Hierarchical regression analysis was performed to examine the effects of optimism and coping flexibility on both perceived stress (Table 2) and life satisfaction (Table 3).

In step 1 , both age and sex were entered as control variables. The results for this step indicated that sex $(P<0.01)$ was a significant predictor of perceived stress, with females reporting higher levels. In step $2 \mathrm{a}$, optimism and coping flexibility were entered together as predictors. It revealed that 34\% $(P<0.001)$ of the variance of perceived stress and $47 \%(P<0.001)$ of the variance of life satisfaction could be explained by the interaction between optimism and coping flexibility. Given the conventional $f^{2}$ effect size values suggested by Cohen ${ }^{43}$ ( small $f^{2}=0.02$; medium $f^{2}=0.15$; large $f^{2}=0.35$ ), both regression analyses reported large effect sizes (effect on perceived stress $f^{2}=0.59$; effect on life satisfaction
Table 2 Hierarchical regression analysis of perceived stress (PSS)

\begin{tabular}{lllll}
\hline Predictors & Step I & Step 2a & Step 2b & Step 3b \\
\cline { 2 - 5 } & $\boldsymbol{\beta}$ & $\boldsymbol{\beta}$ & $\boldsymbol{\beta}$ & $\boldsymbol{\beta}$ \\
\hline Step I & & & & \\
Age & 0.04 & 0.07 & 0.05 & 0.07 \\
Sex & $0.28^{* * *}$ & $0.20^{*}$ & $0.22^{*}$ & $0.20^{*}$ \\
Step 2a & & & & \\
CFS & & -0.12 & & \\
LOT-R & & $-0.53^{* * *}$ & & \\
Step 2b & & & & \\
CFS & & & $-0.35^{* * * *}$ & -0.12 \\
Step 3b & & & & \\
LOT-R & & & & $-0.53^{* * * *}$ \\
$R^{2}$ & 0.08 & 0.42 & 0.20 & 0.42 \\
$\Delta R^{2}$ & $0.08^{*}$ & $0.34^{* * *}$ & $0.12^{* * * *}$ & $0.22^{* * *}$ \\
Model F & $3.95^{*}$ & $16.17^{* * *}$ & $7.42^{* * *}$ & $16.17^{* * *}$ \\
\hline N & & &
\end{tabular}

Notes: $\mathrm{N}=95 . * \mathrm{P}<0.05, * * \mathrm{P}<0.01, * * * \mathrm{P}<0.001$. ${ }^{a}$ Sex: male $=1$ and female $=2$. $\Delta R^{2}=R^{2}$ change.

Abbreviations: $\beta$, standardized $\beta$ coefficient; CFS, Coping Flexibility Scale; LOT-R, Life Orientation Test-Revised; PSS, Perceived Stress Scale.

$\left.f^{2}=0.96\right)$. A post hoc power analysis was conducted using the software package, GPower 3.1. ${ }^{44}$ A sample size of 95 was used for the statistical power analyses, and a four-predictor variable equation was used as a baseline. The $\alpha$ level used for this analysis was $P<0.001$. The post hoc analyses revealed the statistical power exceeded 0.99 for both regression analyses. Thus, there was more than adequate power (ie, power $>0.80$ ). This gave support to Hypothesis 3 .

In step $2 \mathrm{~b}$, a stepwise approach was used to regress coping flexibility onto perceived stress and life satisfaction, prior to optimism. Coping flexibility significantly predicted perceived stress $(\beta=-0.35, P<0.001)$ and life satisfaction $(\beta=0.32$, $P<0.01)$. However, in step $3 \mathrm{~b}$, optimism was entered in the

Table 3 Hierarchical regression analysis of life satisfaction (SWLS)

\begin{tabular}{lllll}
\hline Predictors & Step I & Step 2a & Step 2b & Step 3b \\
\cline { 2 - 5 } & $\beta$ & $\beta$ & $\beta$ & $\beta$ \\
\hline Step I & & & & \\
Age & -0.12 & $-0.16^{*}$ & -0.03 & $-0.16^{*}$ \\
Sex & -0.16 & -0.09 & -0.10 & -0.09 \\
Step 2a & & & & \\
CFS & & 0.02 & & \\
LOT-R & & $0.68^{* * *}$ & & \\
Step 2b & & & & 0.02 \\
CFS & & & $0.32^{* * *}$ & \\
Step 3b & & & & $0.68^{* * *}$ \\
LOT-R & & & & 0.11 \\
$R^{2}$ & 0.04 & $0.5 \mathrm{I}$ & 0.14 & $0.37^{* * *}$ \\
$\Delta R^{2}$ & 0.04 & $0.47^{* * *}$ & $0.10^{* *}$ & $23.12^{* * *}$ \\
Model F & 1.84 & $23.12^{* * *}$ & $4.80^{* *}$ & \\
\hline
\end{tabular}

Notes: $\mathrm{N}=95$. $* \mathrm{P}<0.05, * * \mathrm{P}<0.01, * * * \mathrm{P}<0.001$. ${ }^{\text {a Sex: }}$ male $=\mathrm{I}$ and female $=2$. $\Delta R^{2}=R^{2}$ change.

Abbreviations: $\beta$, standardized $\beta$ coefficient; CFS, Coping Flexibility Scale; LOT-R, Life Orientation Test-Revised; SWLS, Satisfaction with Life Scale. 
model and significantly predicted perceived stress $(\beta=-0.53$, $P<0.001)$ and life satisfaction $(\beta=0.68, P<0.001)$, after controlling for coping flexibility, with the effect of coping flexibility no longer being significant. Optimism also explained $22 \%\left(P<0.001 ; f^{2}=0.40\right)$ of the variance of perceived stress and $37 \%\left(P<0.001 ; f^{2}=0.76\right)$ of the variance of life satisfaction. The analyses suggest a full mediation effect of optimism regarding the association of coping flexibility with both perceived stress and life satisfaction.

\section{Structural equation modeling analyses}

SEM was conducted using Amos 23.0 software (IBM Corporation), to further examine the mediating role of optimism between coping flexibility and psychological health. The direct effects reported were bootstrapped with 1,000 resampling iterations. The paths and parameter estimates for the proposed mediation model with perceived stress as the dependent variable are shown in Figure 1, while that with life satisfaction being the dependent variable are shown in Figure 2 . The diagrams indicate that when coping flexibility was entered alone in the model, it significantly predicted a reduction in perceived stress and an increase in life satisfaction. However, when optimism was added to the model, it had the greatest association with both perceived stress and life satisfaction, and the path coefficients from coping flexibility to both dependent variables became non-significant. Therefore, optimism mediated the effect of coping flexibility.

Both models of perceived stress (1) and life satisfaction (2) were also tested for how well they fit to the data. Chisquare value for the overall model fit was non-significant (1) $\chi^{2}(d f=5, \mathrm{~N}=95) 4.05, P=0.54$; (2) $\chi^{2}(d f=5, \mathrm{~N}=95) 4.05$, $P=0.54]$, suggesting a good fit between both hypothesized models and the data. Furthermore, according to SEM procedures, a model is considered to have a good fit to the data when fit indices have a value generally above 0.95 , and the RMSEA has a value lower than $0.05 .{ }^{45}$ Examination of these indices also showed a good fit between the hypothesized model and the data, including the error of approximation (1) RMSEA =0; (2) RMSEA =0] and all model fit indices [eg, (1) $\mathrm{CFI}=1.00, \mathrm{GFI}=0.98$, NFI $=0.95$; (2) $\mathrm{CFI}=1.00$, GFI $=0.98, \mathrm{NFI}=0.96]$.

In sum, optimism mediated the relationship between coping flexibility and both perceived stress and life satisfaction. Hypothesis 4 was therefore supported.

\section{Discussion}

The present research was conducted to test whether coping flexibility is a reliable and valid construct in a UK sample and to subsequently investigate the association between coping flexibility, optimism, and psychological health - measured by perceived stress and life satisfaction. As far as the author is aware, this study is the first to examine the validity and reliability of the English version of the CFS in a Western population and also to investigate the association between optimism and coping flexibility.

The results establish that the CFS was reliable within a UK undergraduate student sample, providing support for the English version of the CFS to be used in Western population samples. However, further analysis of the CFS revealed that the reliability of the evaluation coping subscale was low, and there was a lack of support for the two-factor structure of the CFS. It appeared likely that this was because of two items, which were also the only two reverse-scored items in the CFS. It is possible that either the wording of these items, the English-language translation of the items, or the evaluation coping subscale may be the issue, and future studies using Western samples are therefore needed in order to resolve this.

As predicted, coping flexibility and optimism were seen to be strongly correlated, the first direct evidence of this. They were also both individually strong predictors of perceived stress and life satisfaction, agreeing with findings from previous research. ${ }^{15,17,30}$ The interaction between coping flexibility and optimism predicted a large amount of the variance in

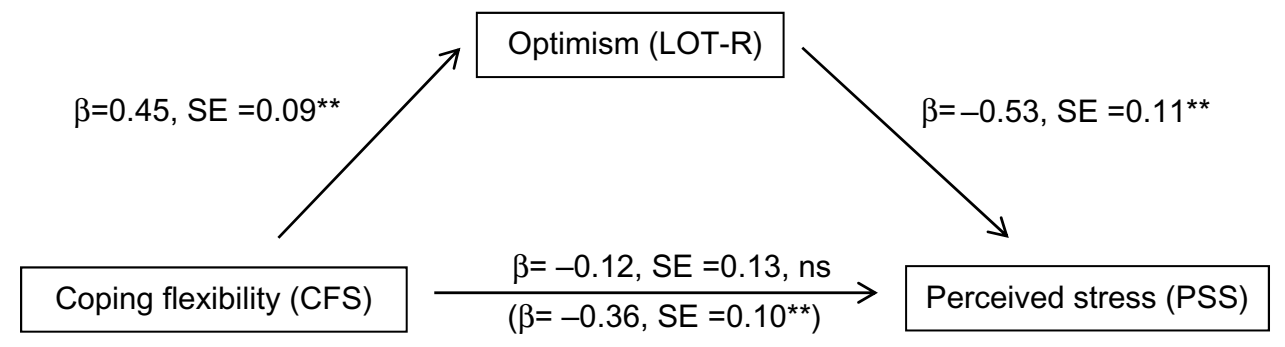

Figure I Effect of coping flexibility on perceived stress, mediated by optimism (sex and age entered as controls but not shown in the diagram). Note: $* * p<0.01$

Abbreviations: $\beta$, standardized $\beta$ coefficient; CFS, Coping Flexibility Scale; LOT-R, Life Orientation Test-Revised; ns, non-significant; PSS, Perceived Stress Scale; $\mathrm{SE}$, standard error. 


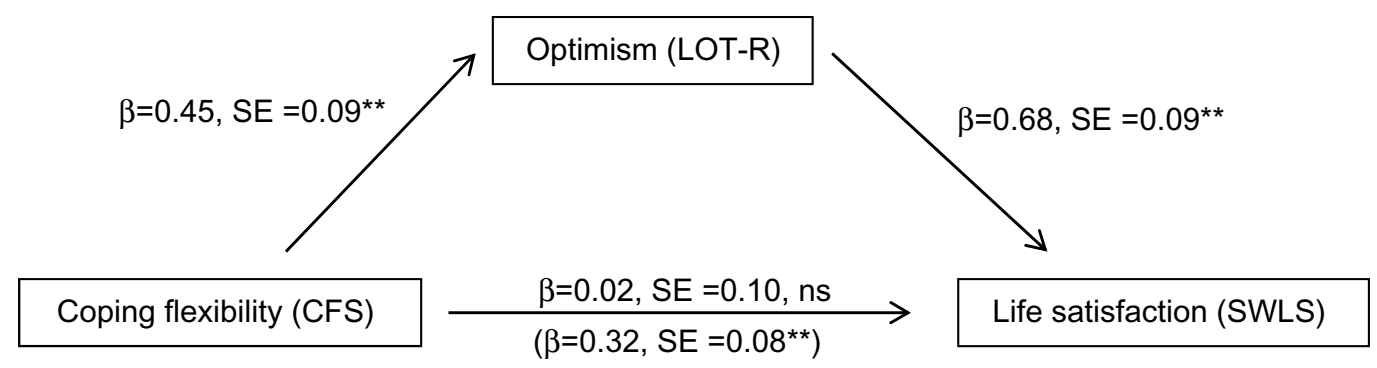

Figure 2 Effect of coping flexibility on life satisfaction, mediated by optimism (sex and age entered as controls but not shown in the diagram). Note: $* * P<0.01$.

Abbreviations: $\beta$, standardized $\beta$ coefficient; CFS, Coping Flexibility Scale; LOT-R, Life Orientation Test-Revised; ns, non-significant; SE, standard error; SWLS, Satisfaction with Life Scale.

both perceived stress and life satisfaction. This is supported by previous evidence suggesting that optimistic individuals will have higher coping flexibility. 13,27,32,34,35 Finally, SEM revealed that optimism mediated the relationship between coping flexibility and both perceived stress and life satisfaction. This suggests that the reason why individuals who cope more flexibly have lower perceived stress and higher life satisfaction is largely explained by their higher levels of reported optimism.

The findings of this study, therefore, add further understanding of what mechanisms are active in allowing people to cope with stress more effectively. Given that optimism appears to have a significant impact in this regard, it would seem logical to target the interventions that help to develop optimism. However, optimism is a personality trait that remains relatively stable over extended periods. Therefore, coping flexibility could provide a more accessible target for interventions that aim at increasing a person's ability to cope with stress. Interventions based on the goodness-of-fit approach to coping flexibility have already been successfully implemented in a health employee sample. ${ }^{14}$ Therefore, stemming from Kato's approach, ${ }^{15}$ interventions could reasonably include using a program aimed at increasing each individual's awareness of the effectiveness of coping strategies and also at enhancing their ability to consider alternative strategies, with the potential to decrease stress and increase feelings of well-being in both workplace and academic environments. The CFS also provides a convenient method to measure the progress of individuals.

Despite the conclusions and overall contribution of this study, the study is limited in the following ways. First, the cross-sectional design limits the causal interpretation of the results. Therefore, it is not possible to determine categorically from the results whether coping flexibility leads to higher levels of optimism or vice versa. Future research could use experimental methods to more stringently test the causal mechanism proposed here. Second, the issues with the CFS limit the statistical strength of the results and, even though the overall scale was found to be reliable, the results should be viewed with caution. Further studies are needed to test the individual items and two-factor structure of the English version of the CFS. Third, the small sample size may have impaired the external consistency of the results, and largerscale studies are needed to confirm the findings. Fourth, the study relied exclusively on self-report measures, which can be open to bias. Nonetheless, the study has provided preliminary insight in this emerging area of research.

\section{Conclusion}

The findings provide preliminary support for coping flexibility and the English version of the CFS to be used in Western populations. However, further analyses of the scale are needed. The findings also add to the occupational stress literature to further our understanding of how optimism is a major factor in psychological health. However, given that optimism is a personality trait, and consequently relatively stable, the study therefore advocates that focus on improving coping flexibility will give the greater potential for achieving sustainable improvement in psychological health through continuous interventions. This will hopefully be of use in affecting beneficial change for the growing numbers of students and employees who report high levels of stress.

\section{Acknowledgments}

The author thanks Seth Gyekye and Catherine Day of Sheffield Hallam University for their helpful support and feedback in preparing the manuscript for publication.

\section{Disclosure}

The author reports no conflicts of interest in this work.

\section{References}

1. Cooper CL, Cartwright S. Healthy mind; healthy organization - a proactive approach to occupational stress. Hum Relat. 1994;47(4):455-471. 
2. Lazarus RS, Folkman S. Stress, Appraisal, and Coping. NY, New York: Springer; 1984.

3. Health and Safety Executive. Stress-related and psychological disorders in Great Britain 2014. Available from: http://www.hse.gov.uk/statistics/ causdis/stress/index.htm. Accessed April 20, 2015.

4. Bewick B, Koutsopoulou G, Miles J, Slaa E, Barkham M. Changes in undergraduate students' psychological well-being as they progress through university. Stud Higher Educ. 2010;35(6):633-645.

5. Bewick BM, Gill J, Mulhearn B, Barkham M, Hill AJ. Using electronic surveying to assess psychological distress within the UK student population: a multi-site pilot investigation. E J Appl Psychol. 2008;4(2):1-5.

6. Skinner EA, Edge K, Altman J, Sherwood H. Searching for the structure of coping: a review and critique of category systems for classifying ways of coping. Psychol Bull. 2003;129(2):216-269.

7. Carver CS, Connor-Smith J. Personality and coping. Annu Rev Psychol. 2010;61:679-704.

8. Lazarus RS. Coping theory and research: past, present, and future. Psychosom Med. 1993;55(3):234-247.

9. Folkman S, Moskowitz JT. Coping: pitfalls and promise. Annu Rev Psychol. 2004;55:745-774.

10. Compas BE, Connor-Smith JK, Saltzman H, Thomsen AH, Wadsworth ME. Coping with stress during childhood and adolescence: problems, progress, and potential in theory and research. Psychol Bull. 2001;127(1):87-127.

11. Bonanno GA, Burton CL. Regulatory flexibility an individual differences perspective on coping and emotion regulation. Perspect Psychol Sci. 2013;8(6):591-612.

12. Cheng $\mathrm{C}$. Assessing coping flexibility in real-life and laboratory settings a multimethod approach. J Pers Soc Psychol. 2001;80(5):814-833.

13. Cheng $\mathrm{C}$, Cheung MW. Cognitive processes underlying coping flexibility: differentiation and integration. J Pers. 2005;73(4):859-886.

14. Cheng C, Kogan A, Chio JH. The effectiveness of a new, coping flexibility intervention as compared with a cognitive-behavioural intervention in managing work stress. Work Stress. 2012;26(3):272-288.

15. Kato T. Development of the coping flexibility scale: evidence for the coping flexibility hypothesis. J Couns Psychol. 2012;59(2):262-273.

16. Folkman S. The case for positive emotions in the stress process. Anxiety Stress Coping. 2008;21(1):3-14.

17. Ng HK, Cheung RY, Tam K. Unraveling the link between narcissism and psychological health: new evidence from coping flexibility. Pers Individ Dif. 2014;70:7-10.

18. Brandtstädter J, Rothermund K. The life-course dynamics of goal pursuit and goal adjustment: a two-process framework. Dev Rev. 2002;22(1):117-150.

19. Nezu AM. Problem solving and behavior therapy revisited. Behav Ther. 2004;35(1):1-33.

20. Carver CS, Scheier MF. Dispositional optimism. Trends Cogn Sci. 2014;18(6):293-299

21. Carver CS, Scheier MF, Segerstrom SC. Optimism. Clin Psychol Rev. 2010;30(7):879-889

22. Seligman M. Learned Optimism. NY, New York: Knopf; 1991.

23. Scheier MF, Carver CS. Optimism, coping, and health: assessment and implications of generalized outcome expectancies. Health Psychol. 1985;4(3):219-247.

24. Scheier MF, Carver CS, Bridges MW. Distinguishing optimism from neuroticism (and trait anxiety, self-mastery, and self-esteem): a reevaluation of the life orientation test. J Pers Soc Psychol. 1994;67(6):1063-1078.

Psychology Research and Behavior Management

\section{Publish your work in this journal}

Psychology Research and Behavior Management is an international, peerreviewed, open access journal focusing on the science of psychology and its application in behavior management to develop improved outcomes in the clinical, educational, sports and business arenas. Specific topics covered include: Neuroscience, memory \& decision making; Behavior
25. Brissette I, Scheier MF, Carver CS. The role of optimism in social network development, coping, and psychological adjustment during a life transition. J Pers Soc Psychol. 2002;82(1):102.

26. De Terte I, Stephens C, Huddleston L. The development of a three part model of psychological resilience. Stress Health. 2014;30(5): 416-424.

27. Nes LS, Segerstrom SC. Dispositional optimism and coping: a metaanalytic review. Pers Soc Psychol Rev. 2006;10(3):235-251.

28. Pavot W, Diener E. Review of the satisfaction with life scale. Psychol Assess. 1993;5(2):164-172.

29. Scheier MF, Carver CS, Bridges MW. Optimism, pessimism, and psychological well-being. In: Chang EC, editor. Optimism and Pessimism: Implications for Theory, Research, and Practice. Washington, DC: American Psychological Association; 2001:189-216.

30. Chang EC. Does dispositional optimism moderate the relation between perceived stress and psychological well-being? A preliminary investigation. Pers Individ Dif. 1998;25(2):233-240.

31. Conversano C, Rotondo A, Lensi E, Della Vista O, Arpone F, Reda MA. Optimism and its impact on mental and physical well-being. Clin Pract Epidemiol Ment Health. 2010;6:25-29.

32. Pavlova MK, Silbereisen RK. Dispositional optimism fosters opportunity congruent coping with occupational uncertainty. J Pers. 2013;81(1):76-86.

33. Foster JD, Trimm RF IV. On being eager and uninhibited: narcissism and approach-avoidance motivation. Pers Soc Psychol Bull. 2008;34(7):1004-1017.

34. Aspinwall LG, MacNamara A. Taking positive changes seriously. Cancer. 2005;104(S11):2549-2556.

35. Galatzer-Levy IR, Burton CL, Bonanno GA. Coping flexibility, potentially traumatic life events, and resilience: a prospective study of college student adjustment. J Soc Clin Psychol. 2012;31(6):542-567.

36. Cohen S, Williamson G. Perceived stress in a probability sample of the United States. In: Spacapam S, Oskamp S, editors. The Social Psychology of Health: Claremont Symposium on Applied Social Psychology. Newbury Park, CA: Sage; 1988.

37. Qualtrics Labs I. Qualtrics Survey Software. Provo, UT: Qualtrics Labs, Inc; 2015.

38. Tabachnick BG, Fidell LS. Using Multivariate Statistics. 5th ed. Boston, MA: Pearson; 2007.

39. Hair JF, Anderson RE, Tatham RL, Black WC. Multivariate Data Analysis with Readings. Upper Saddle River, NJ: Prentice Hall; 1995.

40. Horn JL. A rationale and test for the number of factors in factor analysis. Psychometrika. 1965;30(2):179-185.

41. Cota AA, Longman RS, Holden RR, Fekken GC, Xinaris S. Interpolating 95th percentile eigenvalues from random data: an empirical example. Educ Psychol Meas. 1993;53(3):585-596.

42. Arbuckle JL. Amos 23.0 User's Guide. Chicago: SPSS; 2014.

43. Cohen J. Statistical Power Analysis for the Behavioral Sciences. 2nd ed. Hillsdale, NJ: Lawrence Erlbaum Associates; 1988.

44. Faul F, Erdfelder E, Buchner A, Lang AG. Statistical power analyses using $\mathrm{G}^{*}$ Power 3.1: tests for correlation and regression analyses. Behav Res Methods. 2009;41(4):1149-1160.

45. Schermelleh-Engel K, Moosbrugger H, Müller H. Evaluating the fit of structural equation models: tests of significance and descriptive goodness-of-fit measures. Methods Psychol Res Online. 2003;8(2):23-74.

\section{Dovepress}

modification \& management; Clinical applications; Business \& sports performance management; Social and developmental studies; Animal studies. The manuscript management system is completely online and includes a quick and fair peer-review system. Visit http://www.dovepress. com/testimonials.php to read real quotes from published authors. 